\title{
On the Approximation of a Continuous Function $f(x, y)$ by its Two Dimensional Legendre Wavelet Expansion
}

\author{
Shyam Lal \\ Banaras Hindu University \\ Institute of Science \\ Department of Mathematics \\ Varanasi-221005, India
}

\author{
Vivek Kumar Sharma \\ Banaras Hindu University \\ Institute of Science \\ Department of Mathematics \\ Varanasi-221005, India
}

\begin{abstract}
In this paper, three new estimates of the continuous function $f(x, y)$ by its two dimensional Legendre wavelet expansion have been obtained.These estimators are sharper and best possible in Legendre wavelet analysis.
\end{abstract}

\section{General Terms}

Continuous function, basic wavelet, Wavelet expansion, partial derivatives of a function of two variable, orthogonality of Legendre polynomials, partial sums of Legendre expansions.

\section{Keywords}

Legendre Wavelet, Wavelet Approximation, admissibility condition, Haar Wavelet, functions of bounded derivatives.

\section{INTRODUCTION}

Wavelet Analysis plays an important role in signal processing, Engineering, Technology and Computer applications. The approximation of functions of certain class by trigonometric series is at common places of analysis. Wavelets are new tools to solve partial differential equations and to estimate the approximation of functions. One dimensional Legendre Wavelets are very useful in wavelet analysis. Here,using Legendre polynomials, a new set of two dimensional wavelets are constructed which are known as two dimensional Legendre wavelets. The wavelet approximation of a function $f$ of a single variable using Haar scaling function and Haar wavelet has been determined by several researcher like Natanson [1], Chui [2], Daubechies [3], Meyer [4], Tao [5], in wavelet analysis. Recently Lal and Kumar[6] have determined best wavelet approximation of functions belonging to generalized Lipschitz class. Working in the same direction, Zheng and Wei $|7|$, Chang and Isah $[\overline{8} \mid$ have studied Legendre Wavelet and its applications. But till now no work seems to have been done to obtain wavelet approximation of a function $f(x, y)$ of two variable $x$ and $y$ by two dimensional Legendre wavelet series. The estimators of this paper are new, sharper and best possible in wavelet analysis.

\section{DEFINITIONS}

\subsection{One dimensional Legendre Wavelet}

Wavelets constitute a family of functions constructed from dialation and translation of a single function $\psi \in L^{2}(R)$ called mother wavelet.We write

$$
\psi_{b, a}(x)=|a|^{\frac{-1}{2}} \psi\left(\frac{x-b}{a}\right), a \neq 0 .
$$

If we restrict the values of dialation and translation parameter to $a=a_{0}^{-n}, b=m b_{0} a_{0}{ }^{-n}, a_{0}>1, b_{0}>0$ respectively,the following family of discrete wavelets are constructed:

$$
\psi_{n, m}=\left|a_{0}\right|^{\frac{n}{2}} \psi\left(a_{0}^{n} x-m b_{0}\right)
$$

The one dimensional Legendre wavelet over the interval $[0,1)$ is defined as

$\psi_{n, m}(x)= \begin{cases}\sqrt{m+\frac{1}{2}} 2^{\frac{k}{2}} L_{m}\left(2^{k} x-\hat{n}\right), & \frac{\hat{n}-1}{2^{k}} \leq x<\frac{\hat{n}+1}{2^{k}} \\ 0, & \text { otherwise }\end{cases}$

where $n=1,2, \ldots, 2^{k-1}$ and $m=0,1,2, \ldots M-1$.In above definition,the polynomials $L_{m}$ are Legendre Polynomials of degree $\mathrm{m}$ over the interval $[-1,1]$ which are defined as follows,

$$
\begin{aligned}
& L_{0}(x)=1, \\
& L_{1}(x)=x,
\end{aligned}
$$

$(m+1) L_{m}+1(x)=(2 m+1) x L_{m}(x)-m L_{m-1}(x), \quad m=1,2,3, \ldots$

The set of $\left\{L_{m}(x): m=1,2,3, \ldots\right\}$ in the Hilbert space $L^{2}[-1,1]$ is a complete orthogonal set.Orthogonality of Legendre polynomial on the interval $[-1,1]$ implies that

$$
\left\langle L_{m}(x), L_{n}(x)\right\rangle=\int_{-1}^{1} L_{m}(x) \overline{L_{n}(x)} d x= \begin{cases}\frac{2}{2 m+1}, & m=n ; \\ 0, & \text { otherwise },\end{cases}
$$


Furthermore,the set of wavelets $\psi_{n, m}(x)$ makes an orthonormal basis in $L^{2}[0,1)$, i.e

$$
\int_{0}^{1} \psi_{n, m}(x) \psi_{n^{\prime} m^{\prime}}(x) d x=\delta_{n, n^{\prime}} \delta_{m, m^{\prime}}
$$

in which $\delta$ denotes Kronecker delta function defined by

$$
\delta_{n, n^{\prime}}=\left\{\begin{array}{l}
1, \quad n=n^{\prime} \\
0, \quad \text { otherwise }
\end{array}\right.
$$

The function $f(x) \in L^{2}[0,1)$ is expressed in the Legendre wavelet series as

$$
f(x)=\sum_{n=1}^{\infty} \sum_{m=0}^{\infty} c_{n, m} \psi_{n, m}(x)
$$

where $c_{n, m}=\left\langle f, \psi_{n, m}\right\rangle$. The $\left(2^{k-1}, M\right)^{t h}$ partial sums of above series are given by

$$
S_{2^{k-1}, M}(x)=\sum_{n=1}^{2^{k-1}} \sum_{m=0}^{M-1} c_{n, m} \psi_{n, m}(x) .
$$

\subsection{Two dimensional Legendre wavelet}

One dimensional Legendre wavelets discussed in previous section is now generalized into two dimensional Legendre wavelets as following:

Two dimensional Legendre wavelets over the region $[0,1) \times[0,1)$ can be defined as follows

$$
\begin{aligned}
\psi_{n, m ; n^{\prime}, m^{\prime}}(x, y)= & \psi_{n, m}(x) \psi_{n^{\prime}, m^{\prime}}(y) \\
= & \left\{\begin{array}{lr}
\sqrt{m+\frac{1}{2}} \sqrt{m^{\prime}+\frac{1}{2}} \\
\times 2^{\frac{k+k^{\prime}}{2}} \\
\times L_{m}\left(2^{k} x-\hat{n}\right) \\
\times L_{m^{\prime}}\left(2^{k^{\prime}} y-\hat{n}^{\prime}\right), & \frac{\hat{n}-1}{2^{k}} \leq x<\frac{\hat{n}+1}{2^{k}}, \\
0, & \frac{\hat{n}^{\prime}-1}{2^{k^{\prime}}} \leq y<\frac{\hat{n}^{\prime}+1}{2^{k^{\prime}}} ; \\
0, & \text { otherwise },
\end{array}\right.
\end{aligned}
$$

where $k=1,2, \ldots, k^{\prime}=1,2, \ldots m=0,1,2 \ldots, M-1$, $m^{\prime}=0,1,2 \ldots, M^{\prime}-1, n=1,2 \ldots, 2^{k-1}, n^{\prime}=1,2 \ldots, 2^{k^{\prime}-1}$. By above definition, the region $[0,1) \times[0,1)$ is divided to $2^{k-1} \times 2^{k^{\prime}-1}$ subregions. The paremeter $M$ and $M^{\prime}$ denote the number of Legendre polynomials for variables $x$ and $y$ respectively.So, $M \times M^{\prime}$ wavelets are constructed on each subregions.

\section{LEGENDRE WAVELET EXPANSION}

Any function $f$ in $L^{2}([0,1) \times[0,1)$ can be expanded in double Legendre wavelet series as

$$
f(x, y)=\sum_{n=1}^{\infty} \sum_{n^{\prime}=1}^{\infty} \sum_{m=0}^{\infty} \sum_{m^{\prime}=0}^{\infty} c_{n, m ; n^{\prime}, m^{\prime}} \psi_{n, m ; n^{\prime}, m^{\prime}}(x, y)
$$

Let $S_{2^{k-1}, M ; 2^{k^{\prime}-1}, M^{\prime}}$ denote the $\left(2^{k-1}, M ; 2^{k^{\prime}-1}, M^{\prime}\right)^{t h}$ partial sums of the series 1 , then

$$
\begin{aligned}
S_{2^{k-1}, M ; 2^{k^{\prime}-1}, M^{\prime}}(x, y) & =\sum_{n=1}^{2^{k-1}} \sum_{n^{\prime}=1}^{2^{k^{\prime}-1}} \sum_{m=0}^{M-1} \sum_{m^{\prime}=0}^{M^{\prime}-1}\left(c_{n, m ; n^{\prime}, m^{\prime}}\right. \\
& \left.\times \psi_{n, m ; n^{\prime}, m^{\prime}}(x, y)\right) .
\end{aligned}
$$

\section{LEGENDRE WAVELET APPROXIMATION}

The Legendre Wavelet Approximation $E_{2^{k-1}, M ; 2^{k^{\prime}-1}, M^{\prime}}(f)$ of a function $f \in L^{2}[0,1) \times[0,1)$ is given by

$$
E_{2^{k-1}, M ; 2^{k^{\prime}-1}, M^{\prime}}(f)=\min \left\|f-S_{2^{k-1}, M ; 2^{k^{\prime}-1}, M^{\prime}}\right\|_{2}
$$

where

$$
\|f\|_{2}=\left(\int_{0}^{1} \int_{0}^{1}|f(x, y)|^{2} d x d y\right)^{\frac{1}{2}}
$$

If $E_{2^{k-1}, M ; 2^{k^{\prime}-1}, M^{\prime}}(f) \rightarrow 0$ as $k, k^{\prime}, M, M^{\prime} \rightarrow \infty$ then $E_{2^{k-1}, M ; 2^{k^{\prime}-1}, M^{\prime}}(f)$ is called the best approximation of $f$ of order $\left(2^{k-1}, M ; 2^{k^{\prime}-1}, M^{\prime}\right)$.Zygmund [9]

\section{THEOREMS}

In this paper we prove the following theorems.

\subsection{Theorem}

Let $f(x, y)$ be continuous function on $[0,1) \times[0,1)$ into $R$ such that $0<\left|\frac{\partial^{2} f}{\partial x \partial y}\right|=N_{1}<\infty \forall(x, y) \in[0,1) \times[0,1)$ and the double Legendre Wavelet Series of $f(x, y)$ be

$$
f(x, y)=\sum_{n=1}^{\infty} \sum_{m=0}^{\infty} \sum_{n^{\prime}=1}^{\infty} \sum_{m^{\prime}=0}^{\infty} c_{n, m ; n^{\prime} . m^{\prime}} \psi_{n, m ; n^{\prime}, m^{\prime}}(x, y)
$$

having $\left(2^{k-1}, M ; 2^{k^{\prime}-1}, M^{\prime}\right)^{t h}$ partial sums

$S_{2^{k-1}, M ; 2^{k^{\prime}-1}, M^{\prime}}(x, y)=\sum_{n=1}^{2^{k-1}} \sum_{m=0}^{M-1} \sum_{n^{\prime}=1}^{2^{k^{\prime}-1}} \sum_{m^{\prime}=0}^{M^{\prime}-1} c_{n, m ; n^{\prime}, m^{\prime}} \psi_{n, m ; n^{\prime}, m^{\prime}}(x, y)$

then Legendre Wavelet approximation $E_{2^{k-1}, M ; 2^{k^{\prime}-1}, M^{\prime}}^{(1)}(f)$ of $f(x, y)$ by $S_{2^{k-1}, M ; 2^{k^{\prime}-1}, M^{\prime}}$ satiesfies

$$
E_{2^{k-1}, M ; 2^{k^{\prime}-1}, M^{\prime}}^{(1)}(f)=O\left[\frac{1}{2^{k+k^{\prime}+1}}\left(\left(\frac{1}{2 M-1}\right)^{\frac{1}{2}}+\left(\frac{1}{2 M^{\prime}-1}\right)^{\frac{1}{2}}\right)\right] \text {, }
$$

for $M \geq 1$ and $M^{\prime} \geq 1$.

\subsection{Theorem}

If $f(x, y)$ is continuous function on $[0,1) \times[0,1)$ into $R$ such that $0<\left|\frac{\partial^{4} f}{\partial x^{3} \partial y}\right|=N_{2}<\infty \forall(x, y) \in[0,1) \times[0,1)$. Then Legendre Wavelet approximation $E_{2^{k-1}, M ; 2^{k^{\prime}-1}, M^{\prime}}^{(2)}(f)$ of $f(x, y)$ is given by

$$
\begin{gathered}
E_{2^{k-1}, M ; 2^{k^{\prime}-1}, M^{\prime}}^{(2)}(f)=O\left[\frac{1}{2^{3 k+k^{\prime}+1}}\right. \\
\left.\left(\left(\frac{1}{2 M-5}\right)^{\frac{5}{2}}+\left(\frac{1}{2 M^{\prime}-1}\right)^{\frac{1}{2}}\right)\right] \\
\text { for } M \geq 3 \text { and } M^{\prime} \geq 1 .
\end{gathered}
$$

\subsection{Theorem}

Let $f(x, y)$ be continuous function on $[0,1) \times[0,1)$ into $R$ such that $0<\left|\frac{\partial^{6} f}{\partial x^{3} \partial y^{3}}\right|=N_{3}<\infty \forall(x, y) \in[0,1) \times[0,1)$.Then 
Legendre Wavelet approximation $E_{2^{k-1}, M ; 2^{k^{\prime}-1}, M^{\prime}}^{(3)}(f)$ of $f(x, y)$ satiesfies

$$
\begin{aligned}
& E_{2^{k-1}, M ; 2^{k^{\prime}-1}, M^{\prime}}^{(3)}(f)= O\left[\left(\frac{1}{2^{3 k+3 k^{\prime}+1}}\right)\right. \\
&\left.\times\left(\left(\frac{1}{2 M-5}\right)^{\frac{5}{2}}+\left(\frac{1}{2 M^{\prime}-5}\right)^{\frac{5}{2}}\right)\right], \\
& \quad \text { for } M \geq 3, M^{\prime} \geq 3 .
\end{aligned}
$$

\section{PROOFS}

\subsection{Proof of Theorem 5.1}

The Legendre Wavelet Series of $f(x, y) \in L^{2}[0,1) \times[0,1)$ is

$$
f(x, y)=\sum_{n=1}^{\infty} \sum_{m=0}^{\infty} \sum_{n^{\prime}=1}^{\infty} \sum_{m^{\prime}=0}^{\infty} c_{n, m ; n^{\prime}, m^{\prime}} \psi_{n, m ; n^{\prime}, m^{\prime}}(x, y)
$$

Then,

$$
\begin{aligned}
\left\langle f, \psi_{p, q}\right\rangle & =\sum_{n=1}^{\infty} \sum_{m=0}^{\infty} \sum_{n^{\prime}=1}^{\infty} \sum_{m^{\prime}=0}^{\infty} c_{n, m ; n^{\prime}, m^{\prime}}\left\langle\psi_{n, m ; n^{\prime}, m^{\prime}}, \psi_{p, q}\right\rangle \\
= & \sum_{n^{\prime}=1}^{\infty} \sum_{m^{\prime}=0}^{\infty} c_{p, q ; n^{\prime}, m^{\prime}}\left\langle\psi_{p, q ; n^{\prime}, m^{\prime}}, \psi_{p, q}\right\rangle \\
\left\langle\left\langle f, \psi_{p, q}\right\rangle, \psi_{p^{\prime}, q^{\prime}}\right\rangle & =\sum_{n^{\prime}=1}^{\infty} \sum_{m^{\prime}=0}^{\infty} c_{p, q ; n^{\prime}, m^{\prime}}\left\langle\left\langle\psi_{p, q ; n^{\prime}, m^{\prime}}, \psi_{p, q}\right\rangle, \psi_{p^{\prime}, q^{\prime}}\right\rangle \\
& =c_{p, q ; p^{\prime}, q^{\prime}}\left\langle\left\langle\psi_{p, q ; p^{\prime}, q^{\prime}}, \psi_{p, q}\right\rangle, \psi_{p^{\prime}, q^{\prime}}\right\rangle
\end{aligned}
$$

Thus,

,by orthogonality of $\psi_{n, m ;, n^{\prime}, m^{\prime}}$

$$
\begin{aligned}
c_{p, q ; p^{\prime}, q^{\prime}} & =\frac{\left\langle\left\langle f, \psi_{p, q}\right\rangle, \psi_{p^{\prime}, q^{\prime}}\right\rangle}{\left\langle\left\langle\psi_{p, q ; p^{\prime}, q^{\prime}}, \psi_{p, q}\right\rangle, \psi_{p^{\prime}, q^{\prime}}\right\rangle} \\
& =\frac{\left\langle\left\langle f, \psi_{p, q}\right\rangle, \psi_{p^{\prime}, q^{\prime}}\right\rangle}{\left\langle\psi_{p, q}, \psi_{p, q}\right\rangle\left\langle\psi_{p^{\prime}, q^{\prime}}, \psi_{p^{\prime}, q^{\prime}}\right\rangle}
\end{aligned}
$$

$c_{p, q ; p^{\prime}, q^{\prime}}=\left\langle\left\langle f, \psi_{p, q}\right\rangle, \psi_{p^{\prime}, q^{\prime}}\right\rangle,\left(\left\langle\psi_{p, q}, \psi_{p, q}\right\rangle=\left\langle\psi_{p^{\prime}, q^{\prime}}, \psi_{p^{\prime}, q^{\prime}}\right\rangle=1\right)$

$$
\begin{aligned}
c_{n, m ; n^{\prime}, m^{\prime}} & =\left\langle\left\langle f, \psi_{n, m}\right\rangle, \psi_{n^{\prime}, m^{\prime}}\right\rangle \\
& =\left\langle\left\langle f, \psi_{n^{\prime}, m^{\prime}}\right\rangle, \psi_{n, m}\right\rangle \\
& =\left\langle\psi_{n, m},\left\langle f, \psi_{n^{\prime}, m^{\prime}}\right\rangle\right\rangle \quad(\langle x, y\rangle=\overline{\langle y, x\rangle})
\end{aligned}
$$

Now,

$$
\begin{aligned}
c_{n, m ; n^{\prime}, m^{\prime}} & =\int_{-\infty}^{\infty} \int_{-\infty}^{\infty} f(x, y) \psi_{n, m}(x) \psi_{n^{\prime}, m^{\prime}}(y) d x d y \\
& =\int_{\frac{\hat{n}-1}{2^{k}}}^{\frac{\hat{n}+1}{2^{k}}} \psi_{n, m}(x)\left(\int_{\frac{\hat{n}^{\prime}-1}{2^{k}}}^{\frac{\hat{n}^{\prime}+1}{2^{k}}} f(x, y) \psi_{n^{\prime}, m^{\prime}}(y) d y\right) d x \\
& =\sqrt{m+\frac{1}{2}} \sqrt{m^{\prime}+\frac{1}{2}} 2^{\frac{k+k^{\prime}}{2}}
\end{aligned}
$$

$$
\begin{aligned}
\times & \int_{\frac{\hat{n}-1}{2^{k}}}^{\frac{\hat{n}+1}{2^{k}}} L_{m}\left(2^{k} x-\hat{n}\right) \\
& \left(\int_{\frac{\hat{n}^{\prime}-1}{2^{k^{\prime}}}}^{\frac{\hat{n}^{\prime}+1}{k^{\prime}}} f(x, y) L_{m^{\prime}}\left(2^{k^{\prime}} y-\hat{n}^{\prime}\right) d y\right) d x
\end{aligned}
$$

Let

$$
I_{1}=\int_{\frac{\hat{n}^{\prime}-1}{2^{k^{\prime}}}}^{\frac{\hat{n}^{\prime}+1}{2^{k^{\prime}}}} f(x, y) L_{m^{\prime}}\left(2^{k^{\prime}} y-\hat{n}^{\prime}\right) d y
$$

Then

$$
\begin{aligned}
I_{1} & =\int_{-1}^{1} f\left(x, \frac{v+\hat{n}^{\prime}}{2^{k^{\prime}}}\right) L_{m^{\prime}}(v) \frac{d v}{2^{k^{\prime}}}, \text { taking } 2^{k^{\prime}} y-\hat{n}^{\prime}=v \\
& =\frac{1}{2^{k^{\prime}}} \int_{-1}^{1} f\left(x, \frac{v+\hat{n}^{\prime}}{2^{k^{\prime}}}\right)\left(\frac{L_{m^{\prime}+1}^{\prime}-L_{m^{\prime}-1}^{\prime}}{2^{k^{\prime}}}\right) d v \\
& =\frac{-1}{2^{2 k^{\prime}}\left(2 m^{\prime}+1\right)} \\
& \times \int_{-1}^{1}\left(\frac{\partial f}{\partial v}\left(x, \frac{v+\hat{n}^{\prime}}{2^{k^{\prime}}}\right)\left(L_{m^{\prime}+1}-L_{m^{\prime}-1}\right) d v\right)
\end{aligned}
$$

Let

$$
I_{2}=\int_{\frac{\hat{n}-1}{2^{k}}}^{\frac{\hat{n}+1}{2^{k}}}\left(\frac{\partial f}{\partial v}\left(x, \frac{v+\hat{n}^{\prime}}{2^{k^{\prime}}}\right) L_{m}\left(2^{k} x-\hat{n}\right) d x\right)
$$

Then

$$
\begin{aligned}
I_{2} & =\frac{-1}{2^{2 k}(2 m+1)} \\
& \times \int_{-1}^{1} \frac{\partial^{2} f}{\partial u \partial v}\left(\frac{u+\hat{n}}{2^{k}}, \frac{v+\hat{n}^{\prime}}{2^{k^{\prime}}}\right)\left(L_{m+1}-L_{m-1}\right) d u
\end{aligned}
$$

Write

$$
\text { , taking } 2^{k} x-\hat{n}=u
$$

$P_{m}(u)=L_{m+1}(u)-L_{m-1}(u)$ and $P_{m^{\prime}}(v)=L_{m^{\prime}+1}(v)-L_{m^{\prime}-1}(v)$.

Collecting equation (2)) to [6], we have

$$
\begin{aligned}
c_{n, m ; n^{\prime}, m^{\prime}} & =\frac{1}{\left(2^{\frac{3 k+3 k^{\prime}+2}{2}}\right)(2 m+1)^{\frac{1}{2}}\left(2 m^{\prime}+1\right)^{\frac{1}{2}}} \\
& \times \int_{-1}^{1} \int_{-1}^{1} \frac{\partial^{2} f}{\partial u \partial v} P_{m}(u) P_{m^{\prime}}(v) d u d v
\end{aligned}
$$

Assume

$$
I_{3}=\int_{-1}^{1} \int_{-1}^{1} \frac{\partial^{2} f}{\partial u \partial v}\left(L_{m+1}-L_{m-1}\right)\left(L_{m^{\prime}+1}-L_{m^{\prime}-1}\right) d u d v
$$

Therefore

$$
\begin{aligned}
\left|I_{3}\right|^{2} & \leq\left(\int_{-1}^{1} \int_{-1}^{1}\left|\frac{\partial^{2} f}{\partial u \partial v}\right|^{2} d u d v\right) \\
& \times\left(\int_{-1}^{1} \int_{-1}^{1}\left(L_{m+1}-L_{m-1}\right)^{2}\left(L_{m^{\prime}+1}-L_{m^{\prime}-1}\right)^{2} d u d v\right)
\end{aligned}
$$




$$
\begin{aligned}
& =4 N_{1}^{2}\left(\frac{4}{2 m-1}\right)\left(\frac{4}{2 m^{\prime}-1}\right) \quad m \geq 1, m^{\prime} \geq 1 \\
& =\frac{64 N_{1}^{2}}{(2 m-1)\left(2 m^{\prime}-1\right)}, m \geq 1, m^{\prime} \geq 1
\end{aligned}
$$

By equation (7) and (8),we get

$$
\begin{array}{r}
\left|c_{n, m ; n^{\prime}, m^{\prime}}\right|^{2} \leq \frac{32 N_{1}{ }^{2}}{2^{3 k+3 k^{\prime}}(2 m-1)^{2}\left(2 m^{\prime}-1\right)^{2}}, \\
m \geq 1, m^{\prime} \geq 1 .
\end{array}
$$

Now ,

$$
\begin{aligned}
& f(x, y)-S_{2^{k-1}, M ; 2^{k^{\prime}-1}, M^{\prime}}=\sum_{n=1}^{\infty} \sum_{m=0}^{\infty} \sum_{n^{\prime}=1}^{\infty} \sum_{m^{\prime}=0}^{\infty} c_{n, m ; n^{\prime}, m^{\prime}} \\
& \times \psi_{n, m ; n^{\prime}, m^{\prime}} \\
& -\sum_{n=1}^{2^{k-1}} \sum_{m=0}^{M-1} \sum_{n^{\prime}=1}^{2^{k^{\prime}-1}} \sum_{m^{\prime}=0}^{M^{\prime}-1} c_{n, m ; n^{\prime}, m^{\prime}} \\
& \times \psi_{n, m ; n^{\prime}, m^{\prime}} \\
& =\left(\sum_{n=1}^{2^{k-1}}+\sum_{n=2^{k-1}+1}^{\infty}\right) \\
& \times\left(\sum_{m=0}^{M-1}+\sum_{m=M}^{\infty}\right) \\
& \times\left(\sum_{n^{\prime}=1}^{2^{k^{\prime}-1}}+\sum_{n^{\prime}=2^{k^{\prime}-1}+1}^{\infty}\right) \\
& \times\left(\sum_{m^{\prime}=0}^{M^{\prime}-1}+\sum_{m^{\prime}=M,}^{\infty}\right) c_{n, m ; n^{\prime}, m^{\prime}} \\
& \times \psi_{n, m ; n^{\prime}, m^{\prime}} \\
& -\sum_{n=1}^{2^{k-1}} \sum_{m=0}^{M-1} \sum_{n^{\prime}=1}^{2^{k^{\prime}-1}} \sum_{m^{\prime}=0}^{M^{\prime}-1} c_{n, m ; n^{\prime}, m^{\prime}} \\
& \times \psi_{n, m ; n^{\prime}, m^{\prime}} \\
& =\sum_{n=1}^{2^{k-1}} \sum_{m=0}^{M-1} \sum_{n^{\prime}=1}^{2^{k^{\prime}-1}} \sum_{m^{\prime}=M^{\prime}}^{\infty} c_{n, m ; n^{\prime}, m^{\prime}} \\
& \times \psi_{n, m ; n^{\prime}, m^{\prime}} \\
& +\sum_{n=1}^{2^{k-1}} \sum_{m=M}^{\infty} \sum_{n^{\prime}=1}^{2^{k^{\prime}-1}} \sum_{m^{\prime}=0}^{M^{\prime}-1} c_{n, m ; n^{\prime}, m^{\prime}} \\
& \times \psi_{n, m ; n^{\prime}, m^{\prime}} \\
& +\sum_{n=1}^{2^{k-1}} \sum_{m=M}^{\infty} \sum_{n^{\prime}=1}^{2^{k^{\prime}-1}} \sum_{m=M^{\prime}}^{\infty} c_{n, m ; n^{\prime}, m^{\prime}} \\
& \times \psi_{n, m ; n^{\prime}, m^{\prime}}
\end{aligned}
$$

Then

$$
\left\|f-S_{2^{k-1}, M ; 2^{k^{\prime}-1}, M^{\prime}}\right\|_{2}^{2}=\sum_{n=1}^{2^{k-1}} \sum_{m=0}^{M-1} \sum_{n^{\prime}=1}^{2^{k^{\prime}-1}} \sum_{m^{\prime}=M^{\prime}}^{\infty}\left|c_{n, m ; n^{\prime}, m^{\prime}}\right|^{2}
$$

$$
\begin{aligned}
& +\sum_{n=1}^{2^{k-1}} \sum_{m=M}^{\infty} \sum_{n^{\prime}=1}^{2^{k^{\prime}-1}} \sum_{m^{\prime}=0}^{M^{\prime}-1}\left|c_{n, m ; n^{\prime}, m^{\prime}}\right|^{2} \\
& +\sum_{n=1}^{2^{k-1}} \sum_{m=M}^{\infty} \sum_{n^{\prime}=1}^{2^{k^{\prime}-1}} \sum_{m^{\prime}=M^{\prime}}^{\infty}\left|c_{n, m ; n^{\prime}, m^{\prime}}\right|^{2}
\end{aligned}
$$

$$
\begin{aligned}
& \leq \sum_{n=1}^{2^{k-1}} \sum_{m=0}^{M-1} \sum_{n^{\prime}=1}^{2^{k^{\prime}-1}} \sum_{m^{\prime}=M^{\prime}}^{\infty} \frac{32 N_{1}^{2}}{2^{3 k+3 k^{\prime}}(2 m-1)^{2}\left(2 m^{\prime}-1\right)^{2}} \\
& +\sum_{n=1}^{2^{k-1}} \sum_{m=M}^{\infty} \sum_{n^{\prime}=1}^{2^{k^{\prime}-1}} \sum_{m^{\prime}=0}^{M^{\prime}-1} \frac{32 N_{1}^{2}}{2^{3 k+3 k^{\prime}}(2 m-1)^{2}\left(2 m^{\prime}-1\right)^{2}} \\
& +\sum_{n=1}^{2^{k-1}} \sum_{m=M}^{\infty} \sum_{n^{\prime}=1}^{2^{k^{\prime}-1}} \sum_{m^{\prime}=M^{\prime}}^{\infty} \frac{32 N_{1}^{2}}{2^{3 k+3 k^{\prime}}(2 m-1)^{2}\left(2 m^{\prime}-1\right)^{2}}
\end{aligned}
$$

Since,

$$
\begin{gathered}
\sum_{m=0}^{M-1} \frac{1}{(2 m-1)^{2}}=O\left(1+\frac{1}{(2 M-1)}\right) \\
\sum_{m=M}^{\infty} \frac{1}{(2 m-1)^{2}}=O\left(\frac{1}{(2 M-1)}\right)
\end{gathered}
$$

and

$$
\begin{gathered}
\sum_{m^{\prime}=0}^{M^{\prime}-1} \frac{1}{\left(2 m^{\prime}-1\right)^{2}}=O\left(1+\frac{1}{\left(2 M^{\prime}-1\right)}\right) \\
\sum_{m^{\prime}=M^{\prime}}^{\infty} \frac{1}{\left(2 m^{\prime}-1\right)^{2}}=O\left(\frac{1}{\left(2 M^{\prime}-1\right)}\right)
\end{gathered}
$$

Considering the above expression,

$$
\begin{aligned}
\left\|f-S_{2^{k-1}, M ; 2^{k^{\prime}-1}, M^{\prime}}\right\|_{2}^{2} & \leq \frac{32 N_{1}^{2} C_{1}}{2^{2 k+2 k^{\prime}+2}}\left(1+\frac{1}{(2 M-1)}\right)\left(\frac{1}{\left(2 M^{\prime}-1\right)}\right) \\
& +\frac{32 N_{1}^{2} C_{1}}{2^{2 k+2 k^{\prime}+2}}\left(\frac{1}{(2 M-1)}\right)\left(1+\frac{1}{\left(2 M^{\prime}-1\right)}\right) \\
& +\frac{32 N_{1}^{2} C_{1}}{2^{2 k+2 k^{\prime}+2}}\left(\frac{1}{(2 M-1)}\right)\left(\frac{1}{\left(2 M^{\prime}-1\right)}\right) \\
& =\frac{32 N_{1}^{2} C_{1}}{2^{2 k+2 k^{\prime}+2}}\left[\frac{1}{(2 M-1)}+\frac{1}{\left(2 M^{\prime}-1\right)}\right] \\
& +\frac{32 N_{1}^{2} C_{1}}{2^{2 k+2 k^{\prime}+2}}\left[\frac{3}{(2 M-1)\left(2 M^{\prime}-1\right)}\right] \\
& \leq \frac{32 N_{1}^{2} C_{1}}{2^{2 k+2 k^{\prime}+2}}\left[\frac{1}{(2 M-1)}+\frac{1}{\left(2 M^{\prime}-1\right)}\right] \\
& +\frac{32 N_{1}^{2} C_{1}}{2^{2 k+2 k^{\prime}+2}}\left[\frac{3}{(2 M-1)\left(2 M^{\prime}-1\right)}\right]
\end{aligned}
$$




$$
\begin{aligned}
& \leq \frac{32 N_{1}^{2} C_{1}}{2^{2 k+2 k^{\prime}+2}}\left[\frac{2}{(2 M-1)}+\frac{2}{\left(2 M^{\prime}-1\right)}\right] \\
& +\frac{32 N_{1}^{2} C_{1}}{2^{2 k+2 k^{\prime}+2}}\left[\frac{4}{(2 M-1)\left(2 M^{\prime}-1\right)}\right] \\
& =\frac{64 N_{1}^{2} C_{1}}{2^{2 k+2 k^{\prime}+2}}\left[\frac{1}{(2 M-1)}+\frac{1}{\left(2 M^{\prime}-1\right)}\right] \\
& +\frac{64 N_{1}^{2} C_{1}}{2^{2 k+2 k^{\prime}+2}}\left[\frac{2}{(2 M-1)\left(2 M^{\prime}-1\right)}\right] \\
& \leq \frac{64 N_{1}^{2} C_{1}}{2^{2 k+2 k^{\prime}+2}}\left[\frac{1}{(2 M-1)}+\frac{1}{\left(2 M^{\prime}-1\right)}\right] \\
& +\frac{64 N_{1}^{2} C_{1}}{2^{2 k+2 k^{\prime}+2}}\left[\frac{2}{(2 M-1)^{\frac{1}{2}}\left(2 M^{\prime}-1\right)^{\frac{1}{2}}}\right] \\
& =\frac{64 N_{1}^{2} C_{1}}{2^{2 k+2 k^{\prime}+2}} \\
& \times\left[\frac{1}{(2 M-1)^{\frac{1}{2}}}+\frac{1}{\left(2 M^{\prime}-1\right)^{\frac{1}{2}}}\right]^{2} \\
\left\|f-S_{2^{k-1}, M ; 2^{k^{\prime}-1}, M^{\prime}}\right\|_{2} & =\left(\frac{8 N_{1} \sqrt{C_{1}}}{2^{k+k^{\prime}+1}}\right) \\
& \times\left[\frac{1}{(2 M-1)^{\frac{1}{2}}}+\frac{1}{\left(2 M^{\prime}-1\right)^{\frac{1}{2}}}\right]
\end{aligned}
$$

Hence

$$
E_{2^{k-1}, M ; 2^{k^{\prime}-1}, M^{\prime}}^{(1)}=O\left[\frac{1}{2^{k+k^{\prime}+1}}\left(\frac{1}{(2 M-1)^{\frac{1}{2}}}+\frac{1}{\left(2 M^{\prime}-1\right)^{\frac{1}{2}}}\right)\right] .
$$

Thus the Theorem 5.1 is completely established.

\subsection{Proof of Theorem 5.2}

Under the condition of theorem 5.2 , following the proof of the theorem 5.1

$$
\begin{aligned}
\int_{\frac{\hat{n}-1}{2^{k}}}^{\frac{\hat{n}+1}{2^{k}}} f(x, y) L_{m}\left(2^{k} x-\hat{n}\right) d x & =\left(\frac{-1}{2^{2 k}(2 m+1)}\right) \\
& \times \int_{-1}^{1}\left[\frac{\partial f}{\partial u}\left(\frac{u+\hat{n}}{2^{k}}, y\right)\right. \\
& \left.\times\left(L_{m+1}-L_{m-1}\right) d u\right] \\
& =\left(\frac{1}{2^{2 k}(2 m+1)}\right) \\
& \times \int_{-1}^{1}\left[\frac{\partial f}{\partial u}\left(\frac{u+\hat{n}}{2^{k}}, y\right)\right. \\
& \left.\times\left(\frac{L_{m}^{\prime}-L_{m-2}^{\prime}}{2 m-1}\right) d u\right] \\
& -\left(\frac{1}{2^{2 k}(2 m+1)}\right) \\
& \times \int_{-1}^{1}\left[\frac{\partial f}{\partial u}\left(\frac{u+\hat{n}}{2^{k}}, y\right)\right. \\
& \left.\times\left(\frac{L_{m+2}^{\prime}-L_{m}^{\prime}}{2 m+3}\right) d u\right]
\end{aligned}
$$$$
=\left(\frac{1}{2^{2 k}(2 m+1)}\right)
$$$$
\times(0
$$$$
\left.-\int_{-1}^{1} \frac{\partial^{2} f}{\partial u^{2}} \frac{\left(L_{m}-L_{m-2}\right)}{2^{k}(2 m-1)} d u\right)
$$$$
-\frac{1}{2^{2 k}(2 m+1)}
$$$$
\times(0
$$$$
\left.-\int_{-1}^{1} \frac{\partial^{2} f}{\partial u^{2}} \frac{\left(L_{m+2}-L_{m}\right)}{2^{k}(2 m+3)} d u\right)
$$

$=-\frac{1}{2^{2 k}(2 m+1)} \int_{-1}^{1} \frac{\partial^{2} f}{\partial u^{2}} \frac{\left(L_{m}-L_{m-2}\right)}{2^{k}(2 m-1)} d v$

$+\frac{1}{2^{2 k}(2 m+1)} \int_{-1}^{1} \frac{\partial^{2} f}{\partial u^{2}} \frac{\left(L_{m+2}-L_{m}\right)}{2^{k}(2 m+3)} d u$

$=\frac{1}{2^{3 k}(2 m+1)} \int_{-1}^{1} \frac{\partial^{2} f}{\partial u^{2}}\left(\frac{u+\hat{n}}{2^{k}}, y\right) \frac{\left(L_{m+2}-L_{m}\right)}{(2 m+3)} d u$

$-\frac{1}{2^{3 k}(2 m+1)} \int_{-1}^{1} \frac{\partial^{2} f}{\partial u^{2}}\left(\frac{u+\hat{n}}{2^{k}}, y\right) \frac{\left(L_{m}-L_{m-2}\right)}{(2 m-1)} d u$

$=\frac{1}{2^{3 k}(2 m+1)} \int_{-1}^{1} \frac{\partial^{2} f}{\partial u^{2}}\left(\frac{u+\hat{n}}{2^{k}}, y\right) \frac{L_{m+2}}{(2 m+3)} d u$

$-\frac{1}{2^{3 k}(2 m+1)} \int_{-1}^{1} \frac{\partial^{2} f}{\partial u^{2}}\left(\frac{u+\hat{n}}{2^{k}}, y\right) \frac{L_{m}}{(2 m+3)} d u$

$-\frac{1}{2^{3 k}(2 m+1)} \int_{-1}^{1} \frac{\partial^{2} f}{\partial u^{2}}\left(\frac{u+\hat{n}}{2^{k}}, y\right) \frac{L_{m}}{(2 m-1)} d u$

$+\frac{1}{2^{3 k}(2 m-1)} \int_{-1}^{1} \frac{\partial^{2} f}{\partial u^{2}}\left(\frac{u+\hat{n}}{2^{k}}, y\right) \frac{L_{m-2}}{(2 m-1)} d u$

$=\frac{1}{2^{3 k}(2 m+1)} \int_{-1}^{1} \frac{\partial^{2} f}{\partial u^{2}}\left(\frac{u+\hat{n}}{2^{k}}, y\right)\left(\frac{L_{m+3}^{\prime}-L_{m+1}^{\prime}}{(2 m+5)(2 m+3)}\right) d u$

$-\frac{1}{2^{3 k}(2 m+1)} \int_{-1}^{1} \frac{\partial^{2} f}{\partial u^{2}}\left(\frac{u+\hat{n}}{2^{k}}, y\right)\left(\frac{L_{m+1}^{\prime}-L_{m-1}^{\prime}}{(2 m+1)(2 m+3)}\right) d u$

$-\frac{1}{2^{3 k}(2 m+1)} \int_{-1}^{1} \frac{\partial^{2} f}{\partial u^{2}}\left(\frac{u+\hat{n}}{2^{k}}, y\right)\left(\frac{L_{m+1}^{\prime}-L_{m-1}^{\prime}}{(2 m+1)(2 m-1)}\right) d u$

$+\frac{1}{2^{3 k}(2 m+1)} \int_{-1}^{1} \frac{\partial^{2} f}{\partial u^{2}}\left(\frac{u+\hat{n}}{2^{k}}, y\right)\left(\frac{L_{m-1}^{\prime}-L_{m-3}^{\prime}}{(2 m-3)(2 m-1)}\right) d u$

$=\frac{-1}{2^{4 k}(2 m+1)} \int_{-1}^{1} \frac{\partial^{3} f}{\partial v^{3}}\left(\frac{u+\hat{n}}{2^{k}}, y\right)\left(\frac{L_{m+3}-L_{m+1}}{(2 m+5)(2 m+3)}\right) d u$

$+\frac{1}{2^{4 k}(2 m+1)} \int_{-1}^{1} \frac{\partial^{3} f}{\partial v^{3}}\left(\frac{u+\hat{n}}{2^{k}}, y\right)\left(\frac{L_{m+1}-L_{m-1}}{(2 m+1)(2 m+3)}\right) d u$

$+\frac{1}{2^{4 k}(2 m+1)} \int_{-1}^{1} \frac{\partial^{3} f}{\partial u^{3}}\left(\frac{u+\hat{n}}{2^{k}}, y\right)\left(\frac{L_{m+1}-L_{m-1}}{(2 m+1)(2 m-1)}\right) d u$

$-\frac{1}{2^{4 k}(2 m+1)} \int_{-1}^{1} \frac{\partial^{3} f}{\partial v^{3}}\left(\frac{u+\hat{n}}{2^{k}}, y\right)\left(\frac{L_{m-1}-L_{m-3}}{(2 m-3)(2 m-1)}\right) d u$ 


$$
=\frac{1}{2^{4 k}(2 m+1)} \int_{-1}^{1} \frac{\partial^{3} f}{\partial u^{3}}\left(\frac{u+\hat{n}}{2^{k}}, y\right) E_{m}(u) d u .
$$

where

$$
\begin{aligned}
E_{m}(u) & =\frac{L_{m+1}-L_{m+3}}{(2 m+3)(2 m+5)}+2 \frac{\left(L_{m+1}-L_{m-1}\right)}{(2 m-1)(2 m+3)} \\
& +\frac{L_{m-3}-L_{m-1}}{(2 m-1)(2 m-3)}
\end{aligned}
$$

Similarly,

$$
\begin{aligned}
\int_{\frac{\hat{n}^{\prime}-1}{2^{k^{\prime}}}}^{\frac{\hat{n}^{\prime}+1}{2^{\prime}}} f(x, y) L_{m^{\prime}}\left(2^{k^{\prime}} y-\hat{n}^{\prime}\right) d y & =\left(\frac{1}{2^{4 k^{\prime}}\left(2 m^{\prime}+1\right)}\right) \\
& \times \int_{-1}^{1}\left[\frac{\partial^{3} f}{\partial v^{3}}\left(x, \frac{v+\hat{n}^{\prime}}{2^{k^{\prime}}}\right)\right. \\
& \left.\times E_{m^{\prime}}(v) d v\right] . \\
E_{m^{\prime}}(v)=\frac{L_{m^{\prime}+1}-L_{m^{\prime}+3}}{\left(2 m^{\prime}+3\right)\left(2 m^{\prime}+5\right)} & +2 \frac{\left(L_{m^{\prime}+1}-L_{m^{\prime}-1}\right)}{\left(2 m^{\prime}-1\right)\left(2 m^{\prime}+3\right)} \\
+\frac{L_{m^{\prime}-3}-L_{m^{\prime}-1}}{\left(2 m^{\prime}-1\right)\left(2 m^{\prime}-3\right)} &
\end{aligned}
$$

Using equation $2,(4)$ and 10, , we get

$$
\begin{aligned}
c_{n, m ; n^{\prime}, m^{\prime}} & =\left(\frac{1}{2^{\frac{7 k+3 k^{\prime}+2}{2}}(2 m+1)^{\frac{1}{2}}\left(2 m^{\prime}+1\right)^{\frac{1}{2}}}\right) \\
& \times \int_{-1}^{1} \int_{-1}^{1}\left[\frac{\partial^{4} f}{\partial u^{3} \partial v}\left(\frac{u+\hat{n}}{2^{k}}, \frac{v+\hat{n}^{\prime}}{2^{k^{\prime}}}\right)\right. \\
& \left.\times E_{m}(u) P_{m^{\prime}}(v) d u d v\right]
\end{aligned}
$$

Using orthogonal property of Legendre polynomials i.e.

$\int_{-1}^{1} L_{m}^{2}(u) d u=\frac{2}{2 m+1}$ and $\int_{-1}^{1} L_{m}(u) L_{n}(u) d u=0$ for $m \neq n$

$$
\begin{aligned}
\int_{-1}^{1} E_{m}^{2}(u) d u & =\int_{-1}^{1} \frac{L_{m+1}^{2}+L_{m+3}^{2}}{(2 m+3)^{2}(2 m+5)^{2}} d u \\
& +\int_{-1}^{1} \frac{4\left(L_{m+1}^{2}+L_{m-1}^{2}\right)}{(2 m-1)^{2}(2 m+3)^{2}} d u \\
& +\int_{-1}^{1} \frac{L_{m-3}^{2}+L_{m-1}^{2}}{(2 m-1)^{2}(2 m-3)^{2}} d u \\
& =\frac{1}{(2 m+3)^{2}(2 m+5)^{2}}\left(\frac{2}{2 m+3}+\frac{2}{2 m+7}\right) \\
& +\frac{4}{(2 m-1)^{2}(2 m+3)^{2}}\left(\frac{2}{2 m+3}+\frac{2}{2 m-1}\right) \\
& +\frac{1}{(2 m-1)^{2}(2 m-3)^{2}}\left(\frac{2}{2 m-5}+\frac{2}{2 m-1}\right) \\
& \leq \frac{24}{(2 m-5)^{5}}
\end{aligned}
$$

Similarly,

$$
\int_{-1}^{1} P_{m^{\prime}}^{2}(v) d v \leq \frac{4}{\left(2 m^{\prime}-1\right)}
$$

Using Schwarz inequality, equations (13) and (14) in equation (12)

$$
\left|c_{n, m, n^{\prime}, m^{\prime}}\right|^{2} \leq \frac{96 N_{2}^{2}}{2^{7 k+3 k^{\prime}}(2 m-5)^{6}\left(2 m^{\prime}-1\right)^{2}} .
$$

Next,

$$
, m \geq 3, m^{\prime} \geq 1 \text {. }
$$

$\left\|f-S_{2^{k-1}, M ; 2^{k^{\prime}-1}, M^{\prime}}\right\|_{2}^{2}=\sum_{n=1}^{2^{k-1}} \sum_{m=0}^{M-1} \sum_{n^{\prime}=1}^{2^{k^{\prime}-1}} \sum_{m^{\prime}=M^{\prime}}^{\infty}\left|c_{n, m ; n^{\prime}, m^{\prime}}\right|^{2}$

$$
\begin{aligned}
& +\sum_{n=1}^{2^{k-1}} \sum_{m=M}^{\infty} \sum_{n^{\prime}=1}^{2^{k^{\prime}-1}} \sum_{m^{\prime}=0}^{M^{\prime}-1}\left|c_{n, m ; n^{\prime}, m^{\prime}}\right|^{2} \\
& +\sum_{n=1}^{2^{k-1}} \sum_{m=M}^{\infty} \sum_{n^{\prime}=1}^{2^{k^{\prime}-1}} \sum_{m^{\prime}=M^{\prime}}^{\infty}\left|c_{n, m ; n^{\prime}, m^{\prime}}\right|^{2}
\end{aligned}
$$

$\leq \sum_{n=1}^{2^{k-1}} \sum_{m=0}^{M-1} \sum_{n^{\prime}=1}^{2^{k^{\prime}-1}} \sum_{m^{\prime}=M^{\prime}}^{\infty} \frac{96 N_{2}^{2}}{2^{7 k+3 k^{\prime}}(2 m-5)^{6}\left(2 m^{\prime}-1\right)^{2}}$

$+\sum_{n=1}^{2^{k-1}} \sum_{m=M}^{\infty} \sum_{n^{\prime}=1}^{2^{k^{\prime}-1}} \sum_{m^{\prime}=0}^{M^{\prime}-1} \frac{96 N_{2}^{2}}{2^{7 k+3 k^{\prime}}(2 m-5)^{6}\left(2 m^{\prime}-1\right)^{2}}$

$+\sum_{n=1}^{2^{k-1}} \sum_{m=M}^{\infty} \sum_{n^{\prime}=1}^{2^{k^{\prime}-1}} \sum_{m^{\prime}=M^{\prime}}^{\infty} \frac{96 N_{2}^{2}}{2^{7 k+3 k^{\prime}}(2 m-5)^{6}\left(2 m^{\prime}-1\right)^{2}}$

$\leq \frac{96 N_{2}^{2} C_{2}}{2^{6 k+2 k^{\prime}+2}}\left(1+\frac{1}{(2 M-5)^{5}}\right)\left(\frac{1}{\left(2 M^{\prime}-1\right)}\right)$

$+\frac{96 N_{2}^{2} C_{2}}{2^{6 k+2 k^{\prime}+2}}\left(\frac{1}{(2 M-5)^{5}}\right)\left(1+\frac{1}{\left(2 M^{\prime}-1\right)}\right)$

$+\frac{96 N_{2}^{2} C_{2}}{2^{6 k+2 k^{\prime}+2}}\left(\frac{1}{(2 M-5)^{5}}\right)\left(\frac{1}{\left(2 M^{\prime}-1\right)}\right)$

$=\frac{96 N_{2}^{2} C_{2}}{2^{6 k+2 k^{\prime}+2}}\left[\frac{1}{(2 M-5)^{5}}+\frac{1}{\left(2 M^{\prime}-1\right)}+\frac{3}{(2 M-5)^{5}\left(2 M^{\prime}-1\right)}\right]$

,$C_{2}$ being a suitable positive constant.

$\leq \frac{96 N_{2}^{2} C_{2}}{2^{6 k+2 k^{\prime}+2}}\left[\frac{1}{(2 M-5)^{5}}+\frac{1}{\left(2 M^{\prime}-1\right)}+\frac{3}{(2 M-5)^{5}\left(2 M^{\prime}-1\right)}\right]$

$\leq \frac{96 N_{2}^{2} C_{2}}{2^{6 k+2 k^{\prime}+2}}\left[\frac{2}{(2 M-5)^{5}}+\frac{2}{\left(2 M^{\prime}-1\right)}+\frac{4}{(2 M-5)^{5}\left(2 M^{\prime}-1\right)}\right]$

$=\frac{192 N_{2}^{2} C_{2}}{2^{6 k+2 k^{\prime}+2}}\left(\frac{1}{(2 M-5)^{5}}+\frac{1}{\left(2 M^{\prime}-1\right)}+\frac{2}{(2 M-5)^{5}\left(2 M^{\prime}-1\right)}\right)$

$\leq \frac{192 N_{2}^{2} C_{2}}{2^{6 k+2 k^{\prime}+2}}\left[\frac{1}{(2 M-5)^{5}}+\frac{1}{\left(2 M^{\prime}-1\right)}+\frac{2}{(2 M-5)^{\frac{5}{2}}\left(2 M^{\prime}-1\right)^{\frac{1}{2}}}\right]$

$=\frac{192 N_{2}^{2} C_{2}}{2^{6 k+2 k^{\prime}+2}}\left[\frac{1}{(2 M-5)^{5}}+\frac{1}{\left(2 M^{\prime}-1\right)}+\frac{2}{(2 M-5)^{\frac{5}{2}}\left(2 M^{\prime}-1\right)^{\frac{1}{2}}}\right]$

$=\frac{192 N_{2}^{2} C_{2}}{2^{6 k+2 k^{\prime}+2}}\left[\frac{1}{(2 M-5)^{\frac{5}{2}}}+\frac{1}{\left(2 M^{\prime}-1\right)^{\frac{1}{2}}}\right]^{2}, \quad M \geq 3, M^{\prime} \geq 1$.

$\left\|f-S_{2^{k-1}, M ; 2^{k^{\prime}-1}, M^{\prime}}\right\|_{2}=\left[\left(\frac{8 \sqrt{3} N_{2} \sqrt{C_{2}}}{2^{3 k+k^{\prime}+1}}\right)\right.$ 


$$
\left.\times\left(\frac{1}{(2 M-5)^{\frac{5}{2}}}+\frac{1}{\left(2 M^{\prime}-1\right)^{\frac{1}{2}}}\right)\right] .
$$

So,

$E_{2^{k-1}, M ; 2^{k^{\prime}-1}, M^{\prime}}^{(2)}=O\left[\frac{1}{2^{3 k+k^{\prime}+1}}\left(\frac{1}{(2 M-5)^{\frac{5}{2}}}+\frac{1}{\left(2 M^{\prime}-1\right)^{\frac{1}{2}}}\right)\right]$.

Thus the Theorem 5.2 is completely established.

\subsection{Proof of Theorem 5.3}

Now following the proofs of the theorem 5.1 and 5.2 ,

$$
\begin{aligned}
& c_{n, m, n^{\prime}, m^{\prime}}=\left(\frac{1}{2^{\frac{7 k+3 k^{\prime}+2}{2}}} \frac{1}{(2 m+1)^{\frac{1}{2}}} \frac{1}{\left(2 m^{\prime}+1\right)^{\frac{1}{2}}}\right) \\
& \times \int_{-1}^{1} \int_{-1}^{1} \frac{\partial^{4} f}{\partial u^{3} \partial v} E_{m}(u) P_{m^{\prime}}(v) d u d v \\
& =\left(\frac{1}{2^{\frac{7 k+3 k^{\prime}+2}{2}}} \frac{1}{(2 m+1)^{\frac{1}{2}}} \frac{1}{\left(2 m^{\prime}+1\right)^{\frac{1}{2}}}\right) \\
& \times \int_{-1}^{1} \int_{-1}^{1} \frac{\partial^{4} f}{\partial u^{3} \partial v} E_{m}(u)\left(L_{m^{\prime}-1}-L_{m^{\prime}+1}\right) d u d v \\
& =\left(\frac{1}{2^{\frac{7 k+3 k^{\prime}+2}{2}}} \frac{1}{(2 m+1)^{\frac{1}{2}}} \frac{1}{\left(2 m^{\prime}+1\right)^{\frac{1}{2}}}\right) \\
& \times\left\{\int_{-1}^{1} \int_{-1}^{1} \frac{\partial^{4} f}{\partial u^{3} \partial v} E_{m}(u) L_{m^{\prime}-1}(v) d u d v\right\} \\
& -\left(\frac{1}{2^{\frac{7 k+3 k^{\prime}+2}{2}}} \frac{1}{(2 m+1)^{\frac{1}{2}}} \frac{1}{\left(2 m^{\prime}+1\right)^{\frac{1}{2}}}\right) \\
& \times\left\{\int_{-1}^{1} \int_{-1}^{1} \frac{\partial^{4} f}{\partial u^{3} \partial v} E_{m}(u) L_{m^{\prime}+1}(v) d u d v\right\} \\
& =\left(\frac{1}{2^{\frac{7 k+3 k^{\prime}+2}{2}}} \frac{1}{(2 m+1)^{\frac{1}{2}}} \frac{1}{\left(2 m^{\prime}+1\right)^{\frac{1}{2}}}\right) \\
& \times \int_{-1}^{1} \int_{-1}^{1} \frac{\partial^{4} f}{\partial u^{3} \partial v} E_{m}(u)\left(\frac{L_{m^{\prime}}^{\prime}-L_{m^{\prime}-2}^{\prime}}{2 m^{\prime}-1}\right) d u d v \\
& -\left(\frac{1}{2^{\frac{7 k+3 k^{\prime}+2}{2}}} \frac{1}{(2 m+1)^{\frac{1}{2}}} \frac{1}{\left(2 m^{\prime}+1\right)^{\frac{1}{2}}}\right) \\
& \times \int_{-1}^{1} \int_{-1}^{1} \frac{\partial^{4} f}{\partial u^{3} \partial v} E_{m}(u)\left(\frac{L_{m^{\prime}+2}^{\prime}-L_{m^{\prime}}^{\prime}}{2 m^{\prime}+3}\right) d u d v \\
& =\left(\frac{1}{2^{\frac{7 k+3 k^{\prime}+2}{2}}} \frac{1}{(2 m+1)^{\frac{1}{2}}} \frac{1}{\left(2 m^{\prime}+1\right)^{\frac{1}{2}}}\right) \\
& \times[0 \\
& \left.-\int_{-1}^{1} \int_{-1}^{1} \frac{\partial^{5} f}{\partial u^{3} \partial v^{2}} E_{m}(u)\left(\frac{L_{m^{\prime}}-L_{m^{\prime}-2}}{2^{k^{\prime}}\left(2 m^{\prime}-1\right)}\right) d u d v\right] \\
& -\left(\frac{1}{2^{\frac{7 k+3 k^{\prime}+2}{2}}} \frac{1}{(2 m+1)^{\frac{1}{2}}} \frac{1}{\left(2 m^{\prime}+1\right)^{\frac{1}{2}}}\right) \\
& \times[0 \\
& \left.-\int_{-1}^{1} \int_{-1}^{1} \frac{\partial^{5} f}{\partial u^{3} \partial v^{2}} E_{m}(u)\left(\frac{L_{m^{\prime}+2}-L_{m^{\prime}}}{2^{k^{\prime}}\left(2 m^{\prime}+3\right)}\right) d u d v\right]
\end{aligned}
$$

$=\left(\frac{1}{2^{\frac{7 k+5 k^{\prime}+2}{2}}} \frac{1}{(2 m+1)^{\frac{1}{2}}} \frac{1}{\left(2 m^{\prime}+1\right)^{\frac{1}{2}}}\right)$

$\times \int_{-1}^{1} \int_{-1}^{1} \frac{\partial^{5} f}{\partial u^{3} \partial v^{2}} E_{m}(u)\left(\frac{L_{m^{\prime}+2}-L_{m^{\prime}}}{\left(2 m^{\prime}+3\right)}\right) d u d v$

$-\left(\frac{1}{2^{\frac{7 k+5 k^{\prime}+2}{2}}} \frac{1}{(2 m+1)^{\frac{1}{2}}} \frac{1}{\left(2 m^{\prime}+1\right)^{\frac{1}{2}}}\right)$

$\times \int_{-1}^{1} \int_{-1}^{1} \frac{\partial^{5} f}{\partial u^{3} \partial v^{2}} E_{m}(u)\left(\frac{L_{m^{\prime}}-L_{m^{\prime}-2}}{\left(2 m^{\prime}-1\right)}\right) d u d v$

$=\left(\frac{1}{2^{\frac{7 k+5 k^{\prime}+2}{2}}} \frac{1}{(2 m+1)^{\frac{1}{2}}} \frac{1}{\left(2 m^{\prime}+1\right)^{\frac{1}{2}}}\right)$

$\times \int_{-1}^{1} \int_{-1}^{1} \frac{\partial^{5} f}{\partial u^{3} \partial v^{2}} E_{m}(u)\left(\frac{L_{m^{\prime}+2}}{\left(2 m^{\prime}+3\right)}\right) d u d v$

$-\left(\frac{1}{2^{\frac{7 k+5 k^{\prime}+2}{2}}} \frac{1}{(2 m+1)^{\frac{1}{2}}} \frac{1}{\left(2 m^{\prime}+1\right)^{\frac{1}{2}}}\right)$

$\times \int_{-1}^{1} \int_{-1}^{1} \frac{\partial^{5} f}{\partial u^{3} \partial v^{2}} E_{m}(u)\left(\frac{L_{m^{\prime}}}{\left(2 m^{\prime}+3\right)}\right) d u d v$

$-\left(\frac{1}{2^{\frac{7 k+5 k^{\prime}+2}{2}}} \frac{1}{(2 m+1)^{\frac{1}{2}}} \frac{1}{\left(2 m^{\prime}+1\right)^{\frac{1}{2}}}\right)$

$\times \int_{-1}^{1} \int_{-1}^{1} \frac{\partial^{5} f}{\partial u^{3} \partial v^{2}} E_{m}(u)\left(\frac{L_{m^{\prime}}}{\left(2 m^{\prime}-1\right)}\right) d u d v$

$+\left(\frac{1}{2^{\frac{7 k+5 k^{\prime}+2}{2}}} \frac{1}{(2 m+1)^{\frac{1}{2}}} \frac{1}{\left(2 m^{\prime}+1\right)^{\frac{1}{2}}}\right)$

$\times \int_{-1}^{1} \int_{-1}^{1} \frac{\partial^{5} f}{\partial u^{3} \partial v^{2}} E_{m}(u)\left(\frac{L_{m^{\prime}-2}}{\left(2 m^{\prime}-1\right)}\right) d u d v$

$=\left(\frac{1}{2^{\frac{7 k+5 k^{\prime}+2}{2}}} \frac{1}{(2 m+1)^{\frac{1}{2}}} \frac{1}{\left(2 m^{\prime}+1\right)^{\frac{1}{2}}}\right)$

$\times \int_{-1}^{1} \int_{-1}^{1} \frac{\partial^{5} f}{\partial u^{3} \partial v^{2}} E_{m}(u)\left(\frac{L_{m^{\prime}+3}^{\prime}-L_{m^{\prime}+1}^{\prime}}{\left(2 m^{\prime}+3\right)\left(2 m^{\prime}+5\right)}\right) d u d v$

$-\left(\frac{1}{2^{\frac{7 k+5 k^{\prime}+2}{2}}} \frac{1}{(2 m+1)^{\frac{1}{2}}} \frac{1}{\left(2 m^{\prime}+1\right)^{\frac{1}{2}}}\right)$

$\times \int_{-1}^{1} \int_{-1}^{1} \frac{\partial^{5} f}{\partial u^{3} \partial v^{2}} E_{m}(u)\left(\frac{L_{m^{\prime}+1}^{\prime}-L_{m^{\prime}-1}^{\prime}}{\left(2 m^{\prime}+3\right)\left(2 m^{\prime}+1\right)}\right) d u d v$

$-\left(\frac{1}{2^{\frac{7 k+5 k^{\prime}+2}{2}}} \frac{1}{(2 m+1)^{\frac{1}{2}}} \frac{1}{\left(2 m^{\prime}+1\right)^{\frac{1}{2}}}\right)$

$\times \int_{-1}^{1} \int_{-1}^{1} \frac{\partial^{5} f}{\partial u^{3} \partial v^{2}} E_{m}(u)\left(\frac{L_{m^{\prime}+1}^{\prime}-L_{m^{\prime}-1}^{\prime}}{\left(2 m^{\prime}-1\right)\left(2 m^{\prime}+1\right)}\right) d u d v$

$+\left(\frac{1}{2^{\frac{7 k+5 k^{\prime}+2}{2}}} \frac{1}{(2 m+1)^{\frac{1}{2}}} \frac{1}{\left(2 m^{\prime}+1\right)^{\frac{1}{2}}}\right)$

$\times \int_{-1}^{1} \int_{-1}^{1} \frac{\partial^{5} f}{\partial u^{3} \partial v^{2}} E_{m}(u)\left(\frac{L_{m^{\prime}-1}^{\prime}-L_{m^{\prime}-3}^{\prime}}{\left(2 m^{\prime}-1\right)\left(2 m^{\prime}-3\right)}\right) d u d v$

$=\left(\frac{1}{2^{\frac{7 k+5 k^{\prime}+2}{2}}} \frac{1}{(2 m+1)^{\frac{1}{2}}} \frac{1}{\left(2 m^{\prime}+1\right)^{\frac{1}{2}}}\right)$ 
$\times\left[0-\int_{-1}^{1} \int_{-1}^{1} \frac{\partial^{6} f}{\partial u^{3} \partial v^{3}} E_{m}(u)\left(\frac{L_{m^{\prime}+3}-L_{m^{\prime}+1}}{2^{k^{\prime}}\left(2 m^{\prime}+3\right)\left(2 m^{\prime}+5\right)}\right) d u d v\right]$

$-\left(\frac{1}{2^{\frac{7 k+5 k^{\prime}+2}{2}}} \frac{1}{(2 m+1)^{\frac{1}{2}}} \frac{1}{\left(2 m^{\prime}+1\right)^{\frac{1}{2}}}\right)$

$\times\left[0-\int_{-1}^{1} \int_{-1}^{1} \frac{\partial^{6} f}{\partial u^{3} \partial v^{3}} E_{m}(u)\left(\frac{L_{m^{\prime}+1}-L_{m^{\prime}-1}}{2^{k^{\prime}}\left(2 m^{\prime}+3\right)\left(2 m^{\prime}+1\right)}\right) d u d v\right]$

$-\left(\frac{1}{2^{\frac{7 k+5 k^{\prime}+2}{2}}} \frac{1}{(2 m+1)^{\frac{1}{2}}} \frac{1}{\left(2 m^{\prime}+1\right)^{\frac{1}{2}}}\right)$

$\times[0$

$\left.-\int_{-1}^{1} \int_{-1}^{1} \frac{\partial^{6} f}{\partial u^{3} \partial v^{3}} E_{m}(u)\left(\frac{L_{m^{\prime}+1}-L_{m^{\prime}-1}}{2^{k^{\prime}}\left(2 m^{\prime}-1\right)\left(2 m^{\prime}+1\right)}\right) d u d v\right]$

$+\left(\frac{1}{2^{\frac{7 k+5 k^{\prime}+2}{2}}} \frac{1}{(2 m+1)^{\frac{1}{2}}} \frac{1}{\left(2 m^{\prime}+1\right)^{\frac{1}{2}}}\right)$

$\times[0$

$\left.-\int_{-1}^{1} \int_{-1}^{1} \frac{\partial^{6} f}{\partial u^{3} \partial v^{3}} E_{m}(u)\left(\frac{L_{m^{\prime}-1}-L_{m^{\prime}-3}}{2^{k^{\prime}}\left(2 m^{\prime}-1\right)\left(2 m^{\prime}-3\right)}\right) d u d v\right]$

$=\left(-\frac{1}{2^{\frac{7 k+5 k^{\prime}+2}{2}}} \frac{1}{(2 m+1)^{\frac{1}{2}}} \frac{1}{\left(2 m^{\prime}+1\right)^{\frac{1}{2}}}\right)$

$\times \int_{-1}^{1} \int_{-1}^{1} \frac{\partial^{6} f}{\partial u^{3} \partial v^{3}} E_{m}(u)\left(\frac{L_{m^{\prime}+3}-L_{m^{\prime}+1}}{2^{k^{\prime}}\left(2 m^{\prime}+3\right)\left(2 m^{\prime}+5\right)}\right) d u d v$

$+\left(\frac{1}{2^{\frac{7 k+5 k^{\prime}+2}{2}}} \frac{1}{(2 m+1)^{\frac{1}{2}}} \frac{1}{\left(2 m^{\prime}+1\right)^{\frac{1}{2}}}\right)$

$\times \int_{-1}^{1} \int_{-1}^{1} \frac{\partial^{6} f}{\partial u^{3} \partial v^{3}} E_{m}(u)\left(\frac{L_{m^{\prime}+1}-L_{m^{\prime}-1}}{2^{k^{\prime}}\left(2 m^{\prime}+3\right)\left(2 m^{\prime}+1\right)}\right) d u d v$

$+\left(\frac{1}{2^{\frac{7 k+5 k^{\prime}+2}{2}}} \frac{1}{(2 m+1)^{\frac{1}{2}}} \frac{1}{\left(2 m^{\prime}+1\right)^{\frac{1}{2}}}\right)$

$\times \int_{-1}^{1} \int_{-1}^{1} \frac{\partial^{6} f}{\partial u^{3} \partial v^{3}} E_{m}(u)\left(\frac{L_{m^{\prime}+1}-L_{m^{\prime}-1}}{2^{k^{\prime}}\left(2 m^{\prime}-1\right)\left(2 m^{\prime}+1\right)}\right) d u d v$

$-\left(\frac{1}{2^{\frac{7 k+5 k^{\prime}+2}{2}}} \frac{1}{(2 m+1)^{\frac{1}{2}}} \frac{1}{\left(2 m^{\prime}+1\right)^{\frac{1}{2}}}\right)$

$\times \int_{-1}^{1} \int_{-1}^{1} \frac{\partial^{6} f}{\partial u^{3} \partial v^{3}} E_{m}(u)\left(\frac{L_{m^{\prime}-1}-L_{m^{\prime}-3}}{2^{k^{\prime}}\left(2 m^{\prime}-1\right)\left(2 m^{\prime}-3\right)}\right) d u d v$

$=\left(\frac{1}{2^{\frac{7 k+7 k^{\prime}+2}{2}}} \frac{1}{(2 m+1)^{\frac{1}{2}}} \frac{1}{\left(2 m^{\prime}+1\right)^{\frac{1}{2}}}\right)$

$\times \int_{-1}^{1} \int_{-1}^{1} \frac{\partial^{6} f}{\partial u^{3} \partial v^{3}} E_{m}(u) E_{m^{\prime}}(v) d u d v$

Therefore

$$
\left|c_{n, m, n^{\prime}, m^{\prime}}\right|^{2} \leq \frac{576 N_{3}^{2}}{2^{7 k+7 k^{\prime}}(2 m-5)^{6}\left(2 m^{\prime}-5\right)^{6}},
$$

, $m \geq 3, m^{\prime} \geq 3$.

$\left\|f-S_{2^{k-1}, M ; 2^{k^{\prime}-1}, M^{\prime}}\right\|_{2}^{2}=\sum_{n=1}^{2^{k-1}} \sum_{m=0}^{M-1} \sum_{n^{\prime}=1}^{2^{k^{\prime}-1}} \sum_{m^{\prime}=M^{\prime}}^{\infty}\left|c_{n, m ; n^{\prime}, m^{\prime}}\right|^{2}$

$$
\begin{aligned}
& +\sum_{n=1}^{2^{k-1}} \sum_{m=M}^{\infty} \sum_{n^{\prime}=1}^{2^{k^{\prime}-1}} \sum_{m^{\prime}=0}^{M^{\prime}-1}\left|c_{n, m ; n^{\prime}, m^{\prime}}\right|^{2} \\
& +\sum_{n=1}^{2^{k-1}} \sum_{m=M}^{\infty} \sum_{n^{\prime}=1}^{2^{k^{\prime}-1}} \sum_{m^{\prime}=M^{\prime}}^{\infty}\left|c_{n, m ; n^{\prime}, m^{\prime}}\right|^{2}
\end{aligned}
$$

$\leq \sum_{n=1}^{2^{k-1}} \sum_{m=0}^{M-1} \sum_{n^{\prime}=1}^{2^{k^{\prime}-1}} \sum_{m^{\prime}=M^{\prime}}^{\infty} \frac{576 N_{3}^{2}}{2^{7 k+7 k^{\prime}}(2 m-5)^{6}\left(2 m^{\prime}-5\right)^{6}}$

$+\sum_{n=1}^{2^{k-1}} \sum_{m=M}^{\infty} \sum_{n^{\prime}=1}^{2^{k^{\prime}-1}} \sum_{m^{\prime}=0}^{M^{\prime}-1} \frac{576 N_{3}^{2}}{2^{7 k+7 k^{\prime}}(2 m-5)^{6}\left(2 m^{\prime}-5\right)^{6}}$

$+\sum_{n=1}^{2^{k-1}} \sum_{m=M}^{\infty} \sum_{n^{\prime}=1}^{2^{k^{\prime}-1}} \sum_{m^{\prime}=M^{\prime}}^{\infty} \frac{576 N_{3}^{2}}{2^{7 k+7 k^{\prime}}(2 m-5)^{6}\left(2 m^{\prime}-5\right)^{6}}$

$\leq \frac{576 N_{3}^{2} C_{3}}{2^{6 k+6 k^{\prime}+2}}\left(1+\frac{1}{(2 M-5)^{5}}\right)\left(\frac{1}{\left(2 M^{\prime}-5\right)^{5}}\right)$

$+\frac{576 N_{3}^{2} C_{3}}{2^{6 k+6 k^{\prime}+2}}\left(\frac{1}{(2 M-5)^{5}}\right)\left(1+\frac{1}{\left(2 M^{\prime}-5\right)^{5}}\right)$

$+\frac{576 N_{3}^{2} C_{3}}{2^{6 k+6 k^{\prime}+2}}\left(\frac{1}{(2 M-5)^{5}}\right)\left(\frac{1}{\left(2 M^{\prime}-5\right)^{5}}\right)$

$=\frac{576 N_{3}^{2} C_{3}}{2^{6 k+6 k^{\prime}+2}}\left[\frac{1}{(2 M-5)^{5}}+\frac{1}{\left(2 M^{\prime}-5\right)^{5}}+\frac{3}{(2 M-5)^{5}\left(2 M^{\prime}-5\right)^{5}}\right]$ ,$C_{3}$ being a suitable positive constant.

$\leq \frac{576 N_{3}^{2} C_{3}}{2^{6 k+6 k^{\prime}+2}}\left[\frac{1}{(2 M-5)^{5}}+\frac{1}{\left(2 M^{\prime}-5\right)^{5}}+\frac{3}{(2 M-5)^{5}\left(2 M^{\prime}-5\right)^{5}}\right]$

$\leq \frac{576 N_{3}^{2} C_{3}}{2^{6 k+6 k^{\prime}+2}}\left[\frac{2}{(2 M-5)^{5}}+\frac{2}{\left(2 M^{\prime}-5\right)^{5}}+\frac{4}{(2 M-5)^{5}\left(2 M^{\prime}-5\right)^{5}}\right]$

$=\frac{1152 N_{3}^{2} C_{3}}{2^{6 k+6 k^{\prime}+2}}\left(\frac{1}{(2 M-5)^{5}}+\frac{1}{\left(2 M^{\prime}-5\right)^{5}}+\frac{2}{(2 M-5)^{5}\left(2 M^{\prime}-5\right)^{5}}\right)$

$\leq \frac{1152 N_{3}^{2} C_{3}}{2^{6 k+6 k^{\prime}+2}}\left[\frac{1}{(2 M-5)^{5}}+\frac{1}{\left(2 M^{\prime}-5\right)^{5}}+\frac{2}{(2 M-5)^{\frac{5}{2}}\left(2 M^{\prime}-5\right)^{\frac{5}{2}}}\right]$

$=\left(\frac{1152 N_{3}^{2} C_{3}}{2^{6 k+6 k^{\prime}+2}}\right)$

$\times\left[\left(\frac{1}{(2 M-5)^{\frac{5}{2}}}\right)^{2}+\left(\frac{1}{\left(2 M^{\prime}-5\right)^{\frac{5}{2}}}\right)^{2}+\frac{2}{(2 M-5)^{\frac{5}{2}}\left(2 M^{\prime}-5\right)^{\frac{5}{2}}}\right]$ $=\frac{1152 N_{3}^{2} C_{3}}{2^{6 k+6 k^{\prime}+2}}\left(\frac{1}{(2 M-5)^{\frac{5}{2}}}+\frac{1}{\left(2 M^{\prime}-5\right)^{\frac{5}{2}}}\right)^{2}, \quad M \geq 3, M^{\prime} \geq 3$

$$
\begin{aligned}
\left\|f-S_{2^{k-1}, M ; 2^{k^{\prime}-1}, M^{\prime}}\right\|_{2} & =\left(\frac{24 \sqrt{2} N_{3} C_{3}^{\frac{1}{2}}}{2^{3 k+3 k^{\prime}+1}}\right) \\
& \times\left[\frac{1}{(2 M-5)^{\frac{5}{2}}}+\frac{1}{\left(2 M^{\prime}-5\right)^{\frac{5}{2}}}\right]
\end{aligned}
$$

Thus,

$$
E_{2^{k-1}, M ; 2^{k^{\prime}-1}, M^{\prime}}^{(3)}=O\left[\left(\frac{1}{2^{3 k+3 k^{\prime}+1}}\right)\right.
$$




$$
\left.\times\left(\frac{1}{(2 M-5)^{\frac{5}{2}}}+\frac{1}{\left(2 M^{\prime}-5\right)^{\frac{5}{2}}}\right)\right] .
$$

This completes the proof of the Theorem 5.3

\section{CONCLUSION}

(1) The estimates of the Theorem 5.1, 5.2 and 5.3 are

$$
\begin{aligned}
E_{2^{k-1}, M ; 2^{k^{\prime}-1}, M}^{(1)}(f) & =O\left[\left(\frac{1}{2^{k+k^{\prime}+1}}\right)\right. \\
& \left.\times\left(\frac{1}{(2 M-1)^{\frac{1}{2}}}+\frac{1}{\left(2 M^{\prime}-1\right)^{\frac{1}{2}}}\right)\right], \\
E_{2^{k-1}, M ; 2^{k^{\prime}-1, M},}^{(2)}(f) & =O\left[\left(\frac{1}{2^{3 k+k^{\prime}+1}}\right)\right. \\
& \left.\times\left(\frac{1}{(2 M-5)^{\prime} \geq 1}+\frac{1}{\left(2 M^{\prime}-1\right)^{\frac{1}{2}}}\right)\right], \\
E_{2^{k-1}, M ; 2^{k^{\prime}-1}, M,}^{(3)}(f) & =O\left[\left(\frac{1}{2^{3 k+3 k^{\prime}+1}}\right)\right. \\
& \left.\times\left(\frac{1, M^{\prime} \geq 1}{(2 M-5)^{\frac{5}{2}}}+\frac{1}{\left(2 M^{\prime}-5\right)^{\frac{5}{2}}}\right)\right]
\end{aligned}
$$

Since

$$
\begin{aligned}
& E_{2^{k-1}, M ; 2^{k^{\prime}-1}, M}^{(1)}(f) \rightarrow 0, E_{2^{k-1}, M ; 2^{k^{\prime}-1}, M}^{(2)}(f) \rightarrow 0 \\
& \text { and } E_{2^{k-1}, M ; 2^{k^{\prime}-1}, M}^{(3)}(f) \rightarrow 0 \text { as }\left(k, k^{\prime}, M, M^{\prime}\right) \rightarrow \infty
\end{aligned}
$$

therefore these estimates are best approximation in Wavelet analysis.

2. It is observed that estimates of $f(x, y)$ having higher order partial derivatives are more sharper than those function of less partial derivatives.

\section{ACKNOWLEDGEMENTS}

Shyam Lal, one of the authors, is thankful to D.S.T(CIMS) for encouragement to this work.

Vivek Kumar Sharma, one of the authors, is grateful to U.G.C, New Delhi, India for providing financial assistance in the form of Junior Research Fellowship (JRF) vide letter no. 21.06.2015(i)EU - V (Dated - 05/01/2016).

Authors are grateful to the refree for his valuable comments and suggestions which improve the prsentation of this paper.

\section{REFERENCES}

[1] Natanson I. P.(1949), Constructive Function Theory, Gosudarstvennoe Izdatel'stvo Tehniko-Teoreticeskoi Literatury, Moscow .

[2] Chui C. K.(1992), An Introduction to wavelets(Wavelet analysis and its applications), Vol. 1, Academic Press, USA.

[3] Daubechies I.(1992) ,'Ten Lectures on Wavelets" SIAM, Philadelphia, .

[4] Meyer Y.(1993), (Toulouse (1992))(Meyer Y. and Roques S.,eds.), Frontiers, vette Gif-sur-Y, Wavelets their post and their future, Progress in Wavelet analysis applications, pp.9-18.

[5] Tao. T.(1996), On the all most everywhere convergence of wavelet summataion method. Appl. and Comp. Harmonic Anal., 3:384-387.

[6] Lal Shyam and Kumar Susheel (2016), "Best Wavelet Approximation of functions belonging to Generalized Lipschitz Class using Haar Scaling function", Thai Journal of Mathematics, (in press).

[7] Zheng X. and Wei Z. (2016), "Estimates of Approximation Error by Legendre Wavelet", 7, 694-700.

[8] Chang P. and Isah A.(2016), "Legendre Wavelet Operational Matrix of fractional Derivative through wavelet-polynomial transformation and its Applications in Solving Fractional Order Brusselator system", Journal of Physics: Conference Series 693012001.

[9] Zygmund A.(1959), Trigonometric Series Volume I,Cambridge University Press 